\title{
Determination of the elastic properties in CFRP composites: comparison of different approaches based on tensile tests and ultrasonic characterization
}

\author{
Victor Munoz ${ }^{1}$, Marianne Perrin ${ }^{* 2}$, Marie-Laetitia Pastor ${ }^{2}$, Hélène Welemane ${ }^{1}$, \\ Arthur Cantarel ${ }^{2}$ and Moussa Karama ${ }^{1}$ \\ ${ }^{1}$ Université de Toulouse, INP-ENIT, LGP, 47 avenue d'Azereix, 65016 Tarbes, France \\ ${ }^{2}$ Université de Toulouse, IUT, ICA, 1 rue Lautréamont, BP 1624, 65016 Tarbes, France
}

(Received keep as blank , Revised keep as blank , Accepted keep as blank )

\begin{abstract}
The mechanical characterization of composite materials is nowadays a major interest due to their increasing use in the aeronautic industry. The design of most of these materials is based on their stiffness, which is mainly obtained by means of tensile tests with strain gauge measurement. For thin laminated composites, this classical method requires adequate samples with specific orientation and does not provide all the independent elastic constants. Regarding ultrasonic characterization, especially immersion technique, only one specimen is needed and the entire determination of the stiffness tensor is possible. This paper presents a study of different methods to determine the mechanical properties of transversely isotropic carbon fibre composite materials (gauge and correlation strain measurement during tensile tests, ultrasonic immersion technique). Results are compared to ISO standards and manufacturer data to evaluate the accuracy of these techniques.
\end{abstract}

Keywords: carbon fibre composite; elastic constants; ultrasonic characterization; tensile test; stiffness tensor

\section{Introduction}

Fibre-reinforced composites are widely used for many structural applications. The primary benefits of the composite components are the reduction of weight and the simplification of assembly (Soutis 2005). The determination of the mechanical properties is essential for ensuring performance to the composite structures. The determination of their stiffness is critical to provide performance to the structure. In addition, the knowledge of complete elastic stiffness matrix is important for modeling and evaluating the mechanical behavior of composite materials under loading conditions (El Bouazzaoui et al. 1996).

For now, tensile test with strain gauge measurements is the technique normalized by ISO standards to identify composites elastic properties. Such conventional method is destructive in nature and provides only a part of elastic constants when thin plates like laminated composite structures are considered. Accordingly, ultrasonic techniques based on the measurement of

*Corresponding author, Ph.D., E-mail: marianne.perrin@iut-tarbes.fr 
ultrasonic wave velocities provide an interesting and non destructive way to address such issue.

First experimental studies based on ultrasonic technique were done by Zimmer and Cost (1970). They have used transmission contact technique to obtain dynamic elastic stiffness necessary to derive the unidirectional composite elastic behavior. Several ultrasonic bulk wave methods have also been developed to obtain phase velocities in anisotropic plates, especially when only one or two sides of the sample are accessible (Chu et al. 1994, Vishnuvardhan et al. 2007).

Immersion ultrasonic technique represents a particularly suitable method in the case of composite materials with small thickness (Munoz et al. 2014, Reddy et al. 2005). For a transversely isotropic composite, measurements in symmetry planes are sufficient to entirely determine the stiffness tensor (that is the five independents constants) (He and Makeev 2012). Various numerical methods and/or technical devices have then been implemented to interpret experimental results (Balasubramaniam and Whitney 1996, Kawashima et al. 1998).

Generally speaking, two experimental protocols can be used for such immersion technique to get velocities measurements, namely through-transmission and back-reflection techniques (Reddy et al. 2005). Through-transmission method requires two transducers: one to send the wave through the sample and the second one to receive the transmitted wave. As the ultrasonic wave travels through the test sample, the wave is reflected in part as it encounters a medium of different acoustic impedance. Then the transmitted wave is received by the transducer and displayed or stored for analyses.

In the back-reflection technique, a transducer working in pulse/echo mode is associated to a large flat reflector which is positioned parallel to the transmitter. The back-reflected wave travels exactly along the same path as the incident wave in the opposite direction (Rokhlin and Wang 1992). When the sample angle is changed, the position of the incident wave on the back reflector changes. Contrary to through-transmission, it is yet not necessary in this case to move the reflector or the transmitter/receiver transducer due to the large dimensions of the reflector.

The aim of this paper is to compare the back-reflected immersion ultrasonic method with classical mechanical characterizations based on tensile tests. Strain gauges are used to measure the axial and lateral strains and stiffness constants are then estimated from the elastic parts of the stress-strain response. At the same time, a digital image correlation system is also implemented to corroborate the gauge data. Regarding immersion technique, a specific device including a rotation system has been set up to study the response of the material under various incident waves. These three results of stiffness tensor measurement (strain gauges, digital image correlation during tensile tests and ultrasonic characterization) are finally compared and discussed.

\section{Specimen and experimental procedure}

\subsection{Specimen}

In view of its increasing use in aeronautics, a carbon fibre reinforced laminate is considered for this study. The M10R/38\%/UD150/CHS composite is made of 14 unidirectional plies of prepreg leading to a thickness composite $h=2 \mathrm{~mm}$. Sample fabrication is carried out using the manual lay up technique. Then the sample is cured at $125^{\circ} \mathrm{C}$ during 90 minutes at a pressure of 2 bars. The dimensions of the unique sample used for the ultrasonic characterization are $150 \times 100 \times 2 \mathrm{~mm}^{3}$. The density of this composite is measured experimentally, namely $\rho=1449 \mathrm{~kg} / \mathrm{m}^{3}$. This material is representative of a transversely isotropic composite. 


\subsection{Experimental procedure for the tensile test method}

The elastic behavior determination based on mechanical tests requires to apply axial tensile load with different orientation regarding the symmetry axis of the material. In this way, laminated samples with three axis fibre directions $\left(0^{\circ}, 90^{\circ}\right.$ and $45^{\circ}$ ), (see Fig. 1) have been cut according to the standard ISO 527-5 for unidirectional composites (Standard NF EN ISO 527-5 2009). With those tests, only 4 elastics constants can then be determined since loading in the direction of axis 3 (orthogonal to the laminate plane) cannot be done for thin composites plates.

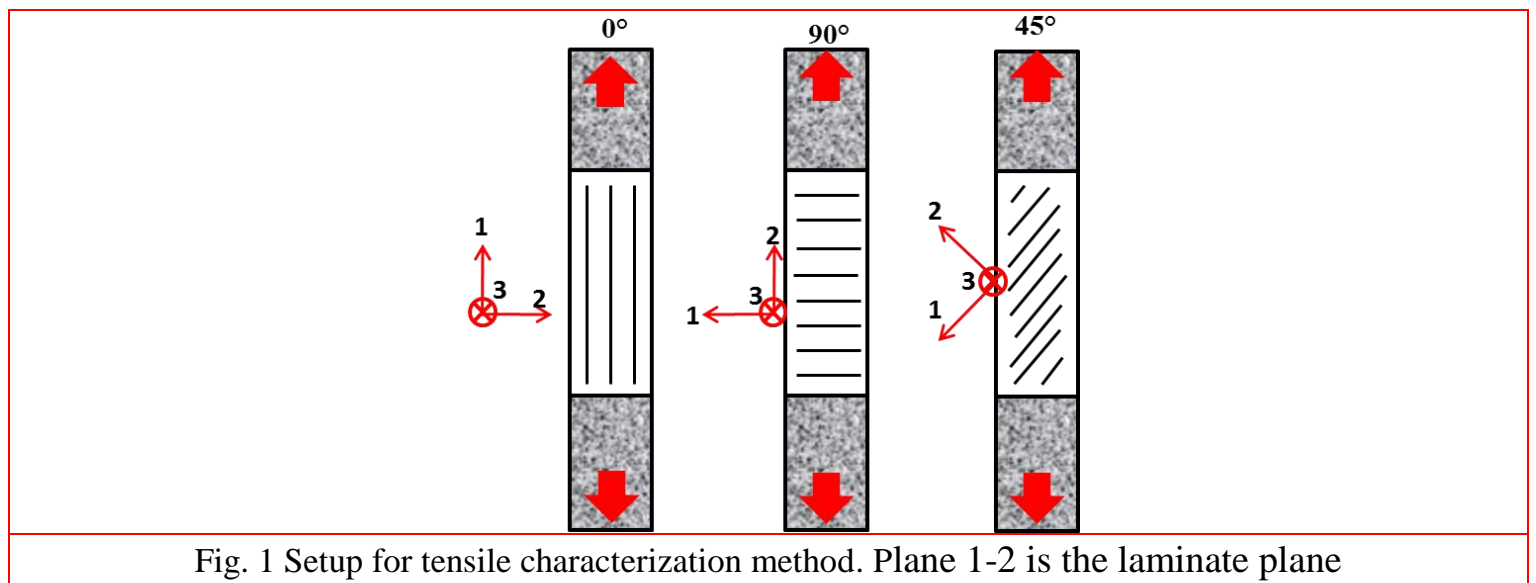

Mechanical tests are carried out at ambient temperature in a room thermoregulated at $25{ }^{\circ} \mathrm{C}$. Uniaxial tensile tests are performed by an electromechanical testing machine INSTRON 5500. According to the standard, the displacement rate is $2 \mathrm{~mm} / \mathrm{min}$ for $0^{\circ}$ samples, $1,5 \mathrm{~mm} / \mathrm{min}$ for $45^{\circ}$ samples and $1 \mathrm{~mm} / \mathrm{min}$ for $90^{\circ}$ samples. To check the reproducibility of the response, three specimens are considered for each loading direction.

Strain gauges of $350 \mathrm{Ohm}$ (HBM K-LY41-6/350-3-2M) are connected to a data acquisition system HBM Spider 8.30 and the data processing is made with Catman Easy software. Digital image correlation system is also performed during tensile tests. Such technique aims at matching two digital images of a surface observed at two different states of load, generally in the reference state (unloaded) and in a deformed state (Sutton et al. 2000).

The markers used for matching the images with the DIC system are done by painting white points on the sample surface (see Fig. 2). The processed image with four markers allows then to obtain the strain during the load for each direction (axial and lateral). The DIC uses an Aramis sensor made of two CCD cameras $1392 \times 1040$ pixels. However for plane structures, only one camera is needed for measuring the 2D strain. These cameras were related to an Aramis software for digital processing. Gauges and monitored markers are positioned on the same face of the specimen. 


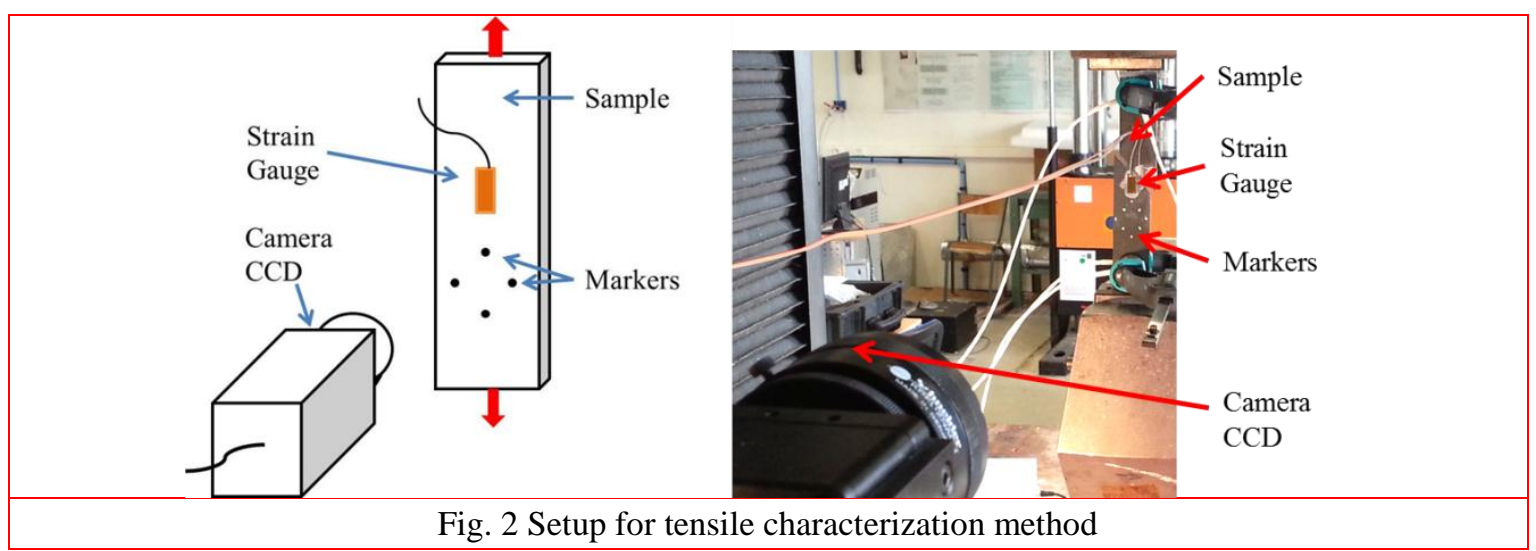

Elastic constants are then calculated for strain states between $P_{R} / 10$ and $P_{R} / 2$ where $P_{R}$ is the yield strength of the specimen. With the $0^{\circ}$ tensile test, Young modulus $E_{1}$ and Poisson ratio $v_{12}$ are classically deduced from the ratios of axial stress and axial and lateral strains. Young modulus $E_{2}=E_{3}$ is calculated in the same way from $90^{\circ}$ tensile test. Finally, Eq. (1) provides the shear modulus $G_{12}$ by calculating Young modulus $E_{45^{\circ}}$ obtained with the $45^{\circ}$ tensile test:

$$
\frac{1}{G_{12}}=\frac{4}{E_{45^{\circ}}}-\frac{1}{E_{1}}-\frac{1}{E_{2}}+2 \frac{\nu_{12}}{E_{1}}
$$

As said before, this procedure does not allow to obtain the last elastic property, namely the Poisson ratio $v_{23}$, that would entirely determine the elastic behavior of the laminated composite.

\subsection{Experimental procedure for ultrasonic characterization}

\subsubsection{General principle}

The elastic stiffness matrix for a transversely isotropic material is given by the following tensor written in the coordinate axes described in Fig. 1:

$$
\left[C_{i j k l}\right]=\left[\begin{array}{cccccc}
C_{11} & C_{12} & C_{12} & 0 & 0 & 0 \\
C_{12} & C_{22} & C_{23} & 0 & 0 & 0 \\
C_{12} & C_{23} & C_{22} & 0 & 0 & 0 \\
0 & 0 & 0 & C_{2323} & 0 & 0 \\
0 & 0 & 0 & 0 & C_{1212} & 0 \\
0 & 0 & 0 & 0 & 0 & C_{1212}
\end{array}\right]
$$

Ultrasonic characterization aims at providing five components representative of the elastic behavior of such material, namely $C_{11}, C_{22}, C_{12}, C_{2323}$ and $C_{1212}\left(C_{23}\right.$ can be derived from $C_{22}$ and $C_{2323}$ ). This determination relies on the resolution of the Christoffel equation (Rose 1999):

$$
|n \cdot C \cdot n-\rho V(n) I|=0
$$

where $V(n)$ is the wave velocity related to the propagation direction of unit vector $n, \rho$ is the material density and $I$ is identity second-order tensor. Accordingly, this requires the measurement of ultrasonic velocities for different orientations of wave propagation in the sample. For a transversely isotropic composite, measurements in planes of symmetry are sufficient to determine 
all five independent elastic constants (Aristégui and Baste 1997, Chu et al. 1994).

For the immersion ultrasonic technique, water acts as a couplant that transfers the wave from the transducer to the sample under inspection (Castagnède et al. 1990, Rokhlin and Wang 1992). The transducer is not directly connected with the sample and hence consistent coupling is ensured. In this way, it is possible to measure the wave velocities at different angles of propagation either by adjusting transducer orientation or by rotating the sample (Reddy et al. 2005). Here the sample is held and moved using a rotation system (see Fig. 3). As ultrasonic generator, an Omniscan 32: $128 \mathrm{PR}$ is used with a mono-element transducer connected to it. The transducer acts at the same time as ultrasonic source and receiver (reflection mode). Classical value of frequency is used, namely $5 \mathrm{MHz}$, which allows mainly to avoid inside reflection; back-reflector and specimen were checked for alignment at normal incidence.

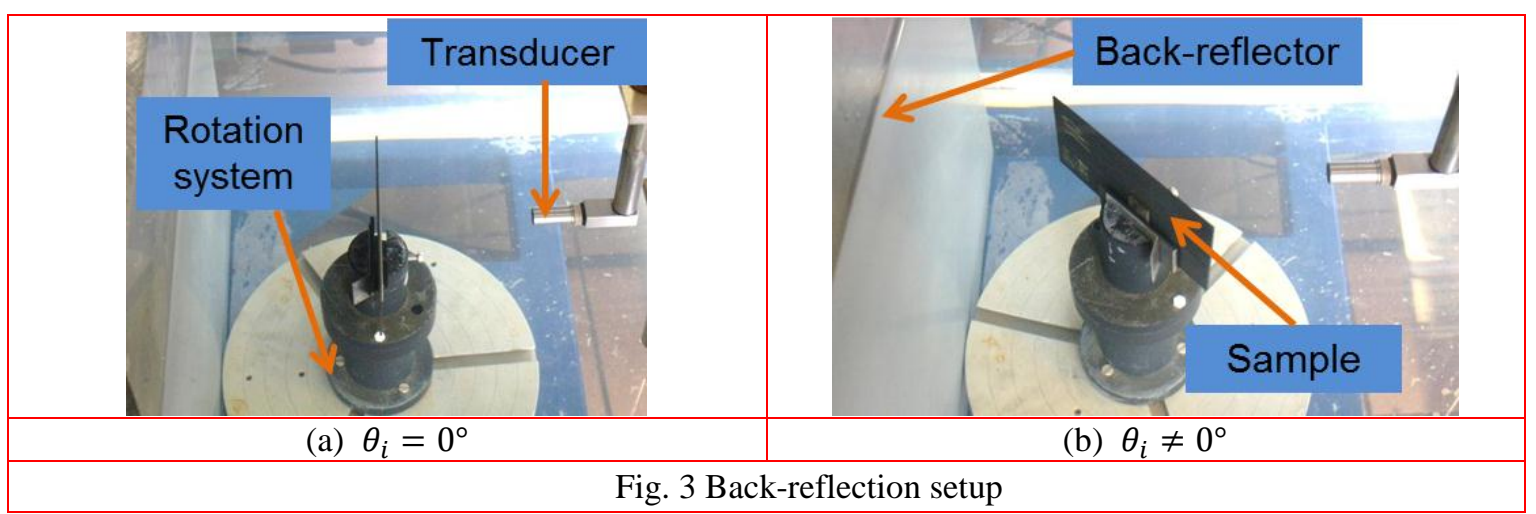

The measurements are performed for different angles $\Phi$ between plane 2-3 and incident plane (see Fig. 4). For a given $\Phi$, the sample is then rotated in the incident plane for different incident angles $\theta_{i}$ between the transducer and axis 3 of the sample. The wave passes through the sample in the direction of $n$ with angle $\theta_{r}$, so $\theta_{r}$ is function of $\Phi$ and $\theta_{i}$. For each set of angles $(\Phi$ and $\theta_{i}$ ), the time of flight is measured.

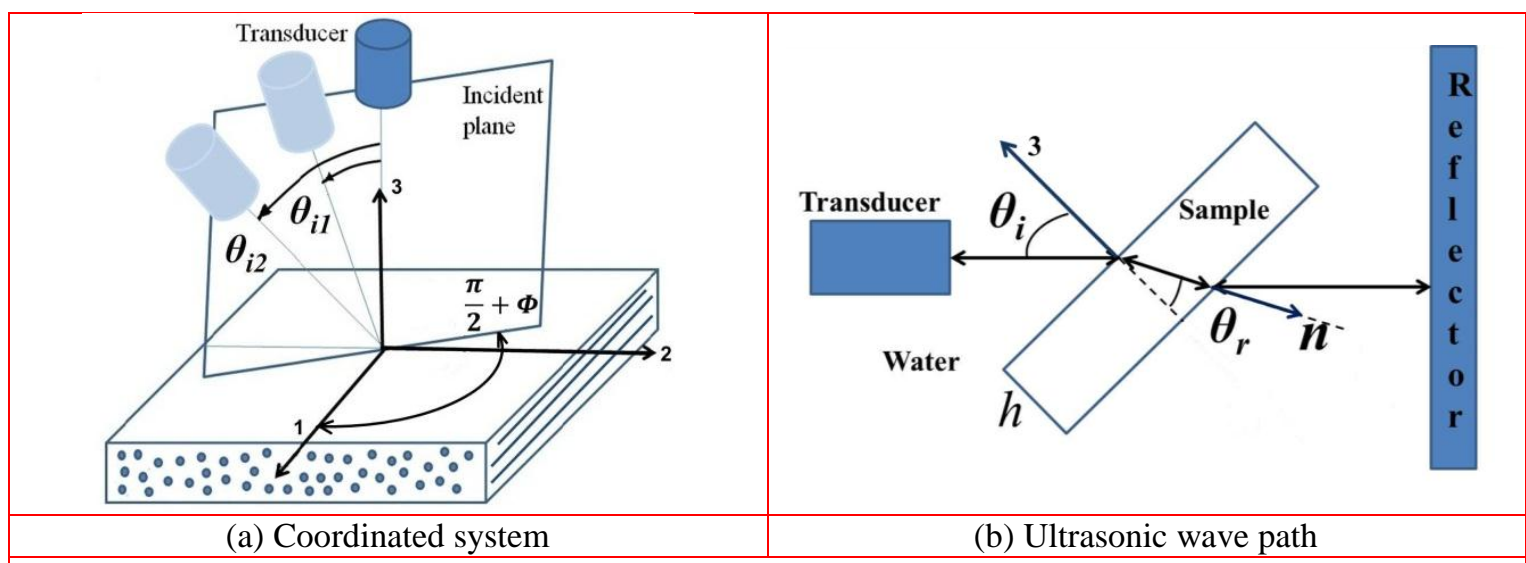

Fig. 4 Wave path description. $\Phi$ : angle between plane 2-3 and incident plan direction. $\theta_{i}$ : incident angle. $\theta_{r}$ : refraction angle 


\subsubsection{Procedure for the elastic constants estimation}

The determination of the velocity of propagation in a medium requires the exact time of travel in the specimen for the given angle of propagation. Such time is determined using the crosscorrelation technique (Rao et al. 1993). It requires a reference signal, which corresponds here to the signal recorded without the sample (only water). The time to cross the material is obtained by subtracting the time of flight along the reference path in the coupling medium (water) to the overall time of flight along the path in the sample at a given incident angle. This time difference $\Delta t$ gives then the ultrasonic velocity $V\left(\theta_{r}\right)$ in the specimen $(\mathrm{m} / \mathrm{s})$ for the refracted angle $\theta_{r}$ by the following relation (Vishnuvardhan et al. 2007):

$$
V(n)=V\left(\Phi, \theta_{i}\right)=\left(\frac{1}{V_{0}^{2}}-\frac{\Delta t \cos \theta_{i}}{h V_{0}}+\frac{(\Delta t)^{2}}{4 h^{2}}\right)^{-1 / 2}
$$

where $V_{0}$ is the velocity in the immersion fluid (water) and $\theta_{i}$ the incidence angle (radians). The transducer senses both longitudinal and quasi-shear waves. Yet, the longitudinal velocity $V_{L}$ of the first one is always more important than the quasi-shear velocity $V_{Q S}$ of the latter; this allows to distinguish their own contribution in the velocity signal (Reddy et al. 2005). These both waves are generated when the incident wave encounters the sample

In the present case, only two configurations of the incident plane are necessary to derive the elastic constants, namely $\Phi=0^{\circ}$ and $\Phi=90^{\circ}$. Fig. 5 shows the ultrasonic velocities obtained in plane 2-3 (that is for $\Phi=0^{\circ}$ ). The longitudinal velocity $V_{L}$ and quasi-shear velocity $V_{Q S}$ are almost constant for all angles of propagation, which stands in agreement with the transverse isotropy of the material around axis 1 . The slight deviation of velocity $V_{L}$ (only $8 \%$ for a range of $28^{\circ}$ of incident angle) can be explained by some misalignment of plies during the manufacturing process. In contrast, regarding the ultrasonic velocities in plane 1-3 (that is for $\Phi=90^{\circ}$, Fig. 6), both longitudinal and shear velocities are clearly affected by the incident angle. This confirms the anisotropic character of the material in this plane (Vishnuvardhan et al. 2007). Note finally that, for incident angles bigger than $60^{\circ}$, no signal can be detected.

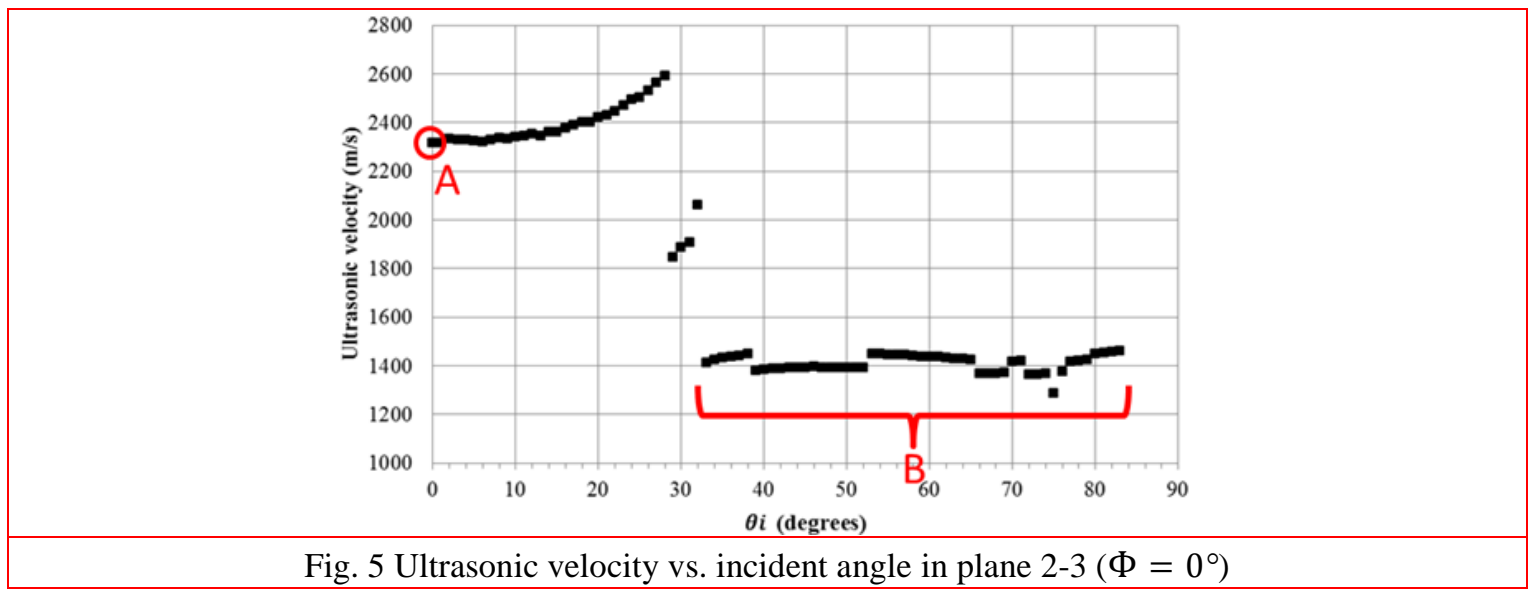




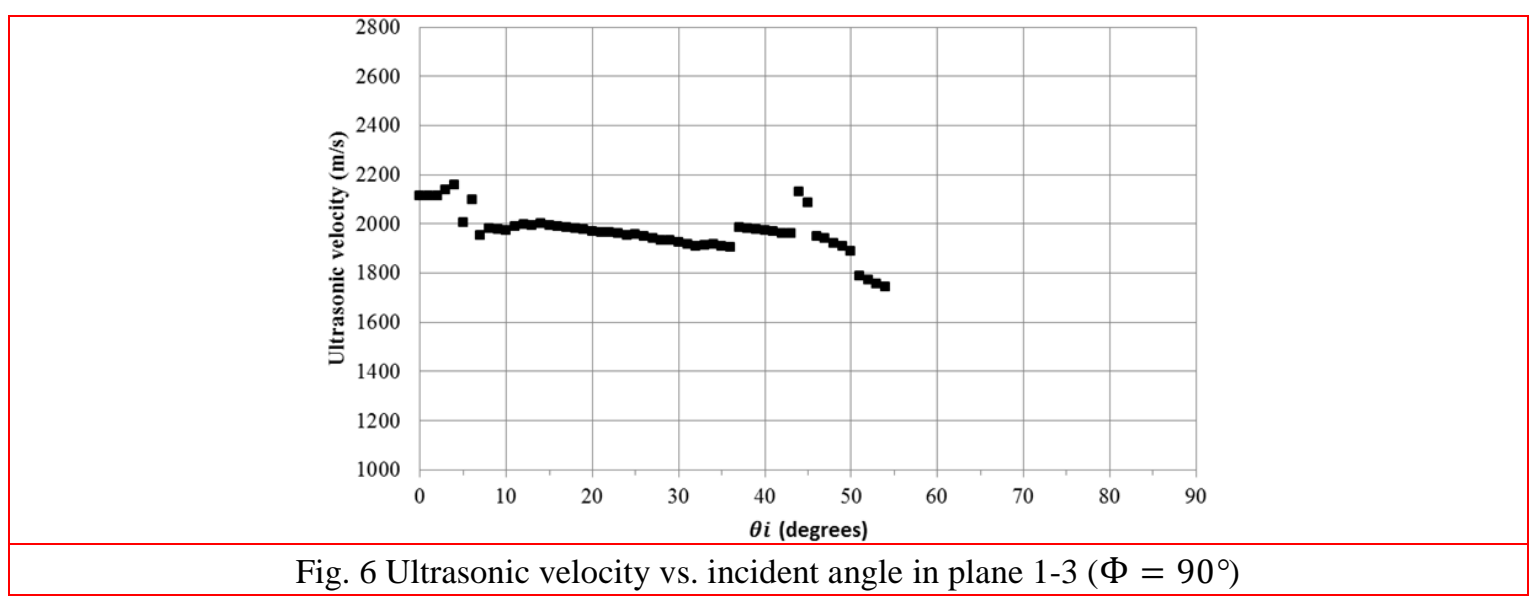

Ultrasonic measurements in plane 2-3 are directly related to the constants $C_{22}$ and $C_{2323}$. Component $C_{22}$ is determined from normal incidence longitudinal velocity, that is for $\theta_{i}=0^{\circ}$ (point A in Fig. 5):

$$
C_{22}=\rho V_{L}^{2}\left(\Phi=0^{\circ}, \theta_{i}=0^{\circ}\right)
$$

On the other hand, $C_{2323}$ is determined from the average shear velocity $V_{Q S}^{\text {mean }}\left(\Phi=0^{\circ}\right)$ data measured in 2-3 plane (part B in Fig. 5):

$$
C_{2323}=\rho\left(V_{Q S}^{\text {mean }}\left(\Phi=0^{\circ}\right)\right)^{2}
$$

$C_{11}, C_{12}$ and $C_{1212}$ are obtained from both longitudinal and shear velocity data measured in 1-3 plane. Experimental velocity data is fitted in the following two relations obtained from the Christoffel equation's solution (Eq. (3)). This allows then to determine the three unknown parameters.

$$
\begin{aligned}
& V_{L}\left(\Phi=90^{\circ}, \theta_{i}\right)=\sqrt{\frac{A+\sqrt{A^{2}-4 B}}{2 \rho}} \\
& V_{Q S}\left(\Phi=90^{\circ}, \theta_{i}\right)=\sqrt{\frac{A-\sqrt{A^{2}-4 B}}{2 \rho}}
\end{aligned}
$$

where

$$
\begin{gathered}
A=\left(C_{12} \cos ^{2} \theta_{i}+C_{11} \sin ^{2} \theta_{i}+C_{1212}\right) \\
B=C_{22} C_{1212} \cos ^{4} \theta_{i}+C_{11} C_{1212} \sin ^{4} \theta_{i}+\frac{\sin ^{2} 2 \theta_{i}}{4}\left[C_{22} C_{11}+C_{1212}^{2}-\left(C_{12}+C_{1212}\right)^{2}\right]
\end{gathered}
$$

To solve undetermined Eqs. (7)-(8), one can use the nonlinear least-square optimization technique which minimizes the deviations between the experimental and theoretical velocities for the considered angles of propagation (Reddy et al. 2005):

$$
\min _{C_{i j k l} \in R^{n}} \frac{1}{2} \sum_{i=1}^{m}\left(V_{i}^{e}-V_{i}^{t}\right)^{2}
$$

In Eq. $11, n$ is the number of independent parameters to be extracted (here 5 elastic constants) 
and $m$ is the number of measurements of velocities in different directions (here 137 experimental data). $V^{e}$ and $V^{t}$ are the experimental and theoretical phase velocities, respectively.

In a last step, component $C_{23}$ is calculated using this relation between elastic stiffness components:

$$
C_{23}=C_{22}-2 C_{2323}
$$

Engineering moduli in the coordinate system $\left(E_{1}, E_{2}=E_{3}, v_{12}, v_{23}\right.$ and $\left.G_{12}\right)$ can be deduced by inverting the stiffness tensor.

\section{Results}

Table 1 and Fig. 7 show the elastic constants estimation obtained by means of the three different methods used to characterize the carbon fibre/epoxy composite. Since tensile test with strain gauge measurements is the technique normalized by ISO standards, results are compared to this method. Indeed, we can see a good accuracy between the $0^{\circ}$ tensile test with strain gauges and the only data provided by the manufacturer, namely the axial Young modulus $E_{1}$.

Table 1 Calculated elastic constants from the three different methods

\begin{tabular}{|c|c|c|c|c|c|c|}
\hline \multicolumn{2}{|c|}{ Test } & $E_{1}[\mathrm{GPa}]$ & $E_{2}[\mathrm{GPa}]$ & $v_{12}$ & $v_{23}$ & $G_{12}[\mathrm{GPa}]$ \\
\hline \multirow{2}{*}{ Tensile test (Gauges) } & Mean & 138 & 8.2 & 0.34 & & 5.1 \\
\cline { 2 - 7 } & $\mathrm{SD}$ & 10 & 1.3 & 0.03 & & 0.3 \\
\hline \multirow{2}{*}{ Tensile test (DIC) } & Mean & 130 & 8.4 & 0.29 & & 4.7 \\
\cline { 2 - 7 } & $\mathrm{SD}$ & 4 & 0.8 & 0.04 & & 0.2 \\
\hline \multirow{2}{*}{ Ultrasonic (US) characterization } & Mean & 123 & 6.4 & 0.42 & 0.13 & 9.0 \\
\cline { 2 - 7 } & $\mathrm{SD}$ & 5 & 1.1 & 0.07 & 0.02 & 0.8 \\
\hline Manufacturer (Hexcel) & Mean & 140 & & & & \\
\hline
\end{tabular}

Regarding DIC technique, we can note a very accurate determination of Young moduli $E_{1}$ (error of $5 \%$ on the mean) and $E_{2}$ (error of $2 \%$ on the mean), whereas results are less precise for Poisson ratio $v_{12}$ (error of $15 \%$ on the mean) and shear modulus $G_{12}$ (error of $8 \%$ on the mean). Latter moduli are determined from lateral strains measurement. For carbon fibre composite subjected to axial tension, these strains exhibit a low amplitude and are then more affected by measurement inaccuracies. However, elastic constants obtained by DIC clearly stand in the range of standard measurements if we consider the standard deviations of each technique. DIC appears then as an interesting alternative to strain gauge, especially as DIC avoids contact and is more practical to set up.

The value of $E_{1}$ in US characterization is the most accurate result in regards to the normalized tensile test (error of $11 \%$ on the mean) and corresponds to a good estimation if the standard deviation is taken into account. Results on mean values of $E_{2}$ (error of $22 \%$ on the mean) and Poisson ratio $v_{12}$ (error of $23 \%$ on the mean) seem less accurate but, again, they stand in the same range as the reference according to the standard deviation of both techniques. The higher error is found for $G_{12}$ (error of $76 \%$ on the mean). All these deviations can be due to the estimation of the time of flight of the wave inside the material. Basically, the time of flight is very 
sensitive to the time reference measured between the transducer and the back-reflector as explained in section 2.3.2. It should be finally underlined that US technique is the single one that provides Poisson ratio $v_{23}$.

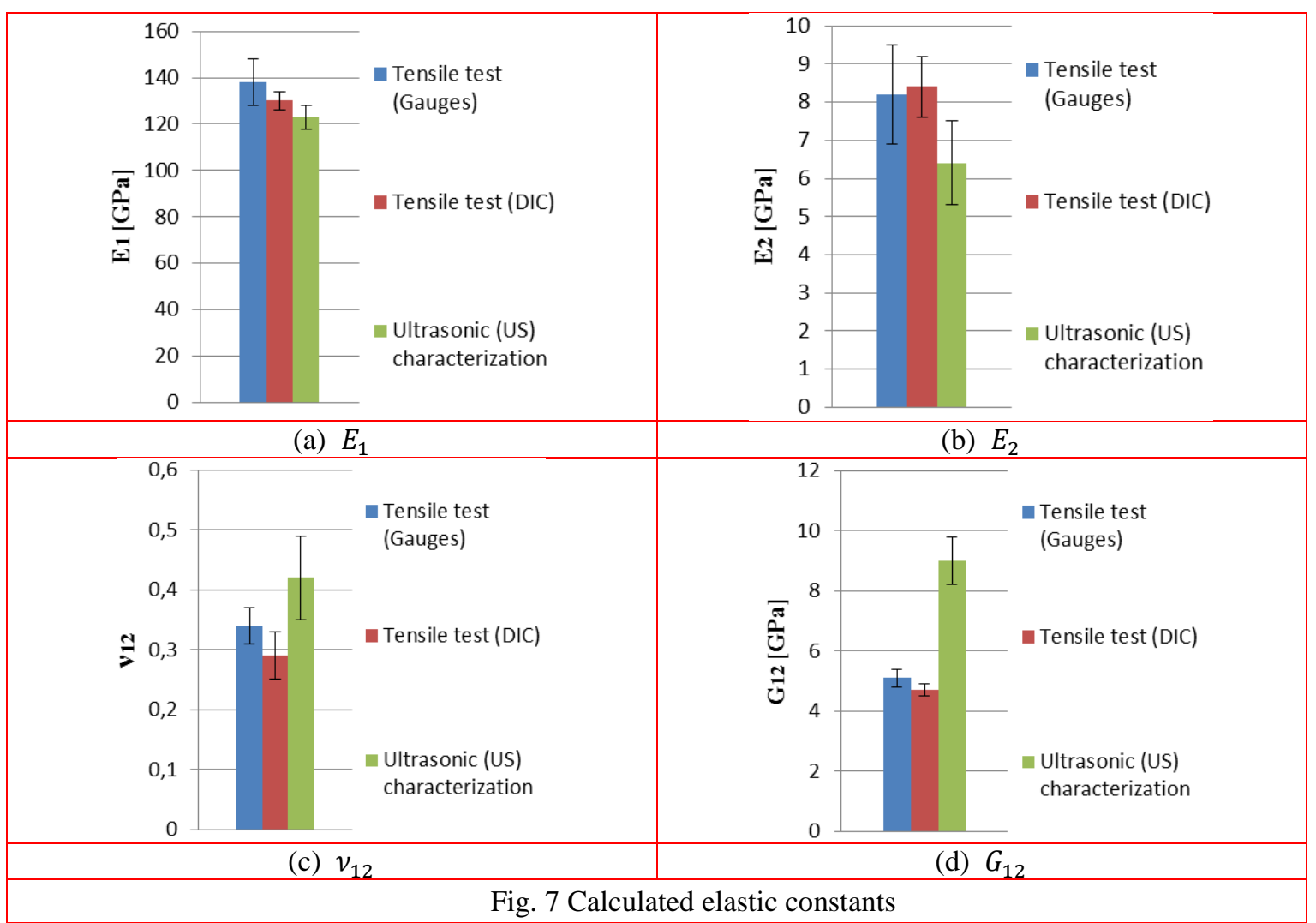

\section{Conclusions}

This paper intended to compare different methodologies to establish the elastic behavior of composite materials. Transversely isotropic composites were considered here through the study of unidirectional carbon fibre/epoxy laminates. Two strain measurement techniques (gauge as standard, Digital Image Correlation) during tensile tests and the immersion ultrasonic method were analyzed.

Results highlight first the interest of DIC to determine the strain response and its good accuracy on the final determination of elastic constants. Yet, tensile tests do not allow to entirely provide the elastic stiffness and some indetermination remains for structures calculation.

This study has shown also the ability of the immersion ultrasonic technique to derive all elastic constants of a UD laminated composite through the measurement of time delay of waves inside a sample. The main advantage of this technique is that only one specimen is needed instead of cutting many samples with desired size and shape for a classical characterization tensile test. If elastic properties estimations are quite encouraging, some limitations on the precision have been 
noted that would require further investigations on the influence of the time of flight. This could be improved by adjusting the experimental setup in such a way that the distance between transducer and reflector is fixed during all the acquisition or by using two transducers (through-transmission).

Given that, this immersion ultrasonic method could then be extended with the same principle to more complex anisotropic materials including orthotropic symmetry. This would need another degree of freedom to rotate the sample that will provide the possibility to propagate ultrasonic waves along more azimuthal angles/planes with respect to the fibre axis.

\section{References}

Aristégui, C. and Baste, S. (1997), "Optimal recovery of the elasticity tensor of general anisotropic materials from ultrasonic velocity data", J. Acoust. Soc. Am., 101(2), 813-833.

Balasubramaniam, K. and Whitney S.C. (1996) "Ultrasonic through-trsmission characterization of thick fiber-reinforced composites", NDT\&E Int., 29(4), 225-236.

Baudouin, S. and Hosten, B. (1996), "Immersion ultrasonic method to measure elastic constants and anisotropic attenuation in polymer-matrix and fiber-reinforced composite materials", Utrasonics, 34, 379382.

Castagnède, B., Jenkins, J.T., Sachse, W. and Baste, S. (1990), "Optimal determination of the elastic constants of composites materials from ultrasonic wavespeed measurements", J. Appl. Phys., 67(4), 27532761.

Chu, Y.C., Degtyar, A.D. and Rokhlin, S.I. (1994), "On determination of orthotropic material moduli from ultrasonic velocity data in nonsymmetry planes", J. Acoust. Soc. Am., 95(6), 3191-3203.

El Bouazzaoui, R., Baste, S. and Camus, G. (1996), "Development of damage in a 2D woven C/SiC composite under mechanical loading: II. Ultrasonic characterization”, Compos. Sci. Technol., 56, 13731382.

He, Y. and Makeev, A. (2012), "Characterization of nonlinear shear properties for composite materials using digital image correlation and finite element analysis", Compos. Sci. Technol., 73, 64-71.

Kawashima, K., Shimizu, Y. and Imuta, M. (1998) "Measurements of anisotropic elastic constants for an aluminium composite plate using a doble reflection method", Review of Progress in Quantitative Nondestructive Evaluation, 17, 1125-1130.

Munoz, V., Perrin, M., Pastor, M.L., Welemane, H., Cantarel, A. and Karama, M. (2014), "Indetification of the elastic properties in CFRP composites", Proceedings of the 5th International Symposium on Aircraft Materials, Marrakech, Morocco, April.

Rao, B.P.C., Jayakumar, T., Bhattacharya, D.B. and Raj, B. (1993), "New methodology for precise ultrasonic velocity measurement and its applications", J. Pure Appl. Ultrason., 15, 53-59.

Reddy, S.S.S., Balasubramaniam, K., Krishnamurthy, C.V. and Shankar M. (2005), "Ultrasonic goniometry immersion techniques for the measurement of elastic moduli", Compos. Struct., 67, 3-17.

Rokhlin, S.I. and Wang, W. (1992), "Double through-transmission bulk wave method for ultrasonic phase velocity measurement and determination of elastic constants of composite materials", J. Acoust. Soc. Am., 91(6), 3303-3312.

Rose, J.L. (1999), Ultrasonic waves in solid media, Cambridge University Press, UK.

Soutis, C. (2005), "Fibre reinforced composites in aircraft construction", Prog. Aerosp. Sci., 41, 143-151.

Sutton, M., McNeill, S.S., Helm, J. and Caho, Y. (2000), "Advances in two-dimensional and threedimensional computer vision”, Photomech. Topics. Apll. Phys., 77, 323-372.

Standard NF EN ISO 527-5 (2009), "Plastics - Determination of tensile properties - Part 5: Test conditions for unidirectional fibre-reinforced plastic composites", AFNOR.

Vishnuvardham, J., Krishnamurthy, C.V. and Balasubramaniam, K. (2007), "Genetic algorithm reconstruction of orthotropic composite plate elastic constants from a single non-symmetric plane 
ultrasonic velocity data", Compos. Part B-Eng., 38, 216-227.

Zimmer, J.E. and Cost, J.R. (1970), "Determination of elastic constants of a unidirectional fiber composite using ultrasonic velocity measurements", J. Acoust. Soc. Am., 47, 795-803. 